\title{
Severity-dependent variations of the neutrophil to lymphocyte ratio (NLR) in peripheral vestibular vertigo
}

\author{
Andi Kurnia Bintang ${ }^{*}$ (i) and Eva lin Magasingan
}

\begin{abstract} the Dizziness Handicap Inventory (DHI) questionnaire. categories were $2.47 \pm 1.66,2.64 \pm 0.96$, and $5.15 \pm 2.59$ respectively. biomarkers in vertigo pathophysiology and clinical assessment.

\section{Introduction}

Vertigo is a phantasm of movement, mostly that of whirling motion, though patients may describe it as a feeling of linear movement or tilt [1]. Peripheral vestibular vertigo is induced by abnormalities of the peripheral vestibular system, semicircular canals, and vestibular nerves. It is mostly caused by dislodging otoconia, endolymphatic hydrops, inner ear viral infection, or trauma of the bony labyrinth or the oval and round window. Vertigo can present as a collection of symptoms consisting of dizziness, nausea, vomiting, nystagmus and unsteadiness.
\end{abstract}

Background: Peripheral vestibular vertigo is a common cause of vertigo especially in the elderly. The neutrophil to lymphocyte ratio (NLR) is a rapid and cost-effective inflammatory marker that has been assessed previously in peripheral vestibular disorders. However, its relation to the severity of peripheral vertigo has not been previously investigated. The aim of this study is to assess the levels of NLR in peripheral vestibular vertigo of various severity categories. This was a cross-sectional study at the Wahidin Sudirohusodo Hospital and Hasanuddin University Teaching Hospital in Makassar. The NLR was measured among subjects with periphel vestibular vertigo with severity categorised using

Results: A total of 39 participants with peripheral vestibular vertigo were recruited. A statistically significant difference was found ( $p=0.002$, Kruskal-Wallis ANOVA) between the mean NLR for the mild, moderate, and severe DHI

Conclusion: A significant difference in the NLR was found between the three different vertigo severity groups, wherein NLR values rise with increase in vertigo severity. This warrants further exploration on the role of inflammatory

Keywords: Inflammation, Neutrophil to lymphocyte ratio, Peripheral vestibular vertigo

\footnotetext{
${ }^{*}$ Correspondence: a.kurnia_b@yahoo.co.id

Department of Neurology, Medical Faculty, Hasanuddin University

Hospital Makassar, Hasanuddin University, Level 4, Building A,

Makassar 90245, Indonesia
}

Complaints of vertigo affect about $20-30 \%$ of the general population, and is more likely to affect females [2]. Vertigo is more frequent in the elderly relative to younger age groups and is the most common complaint in people over 75 years [3]. About $80 \%$ of people who experience vertigo report that the complaint interferes with their daily activities, as it impedes with the ability to work, and results in an increased risk of falling and the high use of health services with downstream impact on economic costs [4].

The severity index of vertigo is often measured by the intensity and frequency of the vertigo attacks. However, severity is a multidimensional concept that may not be fully explained solely by the intensity and frequency of symptoms. Emotional, functional and physical disturbances caused by vertigo can be assessed by the Dizziness Handicap Inventory (DHI) score [5]. In 2004, 
Whitney et al. conducted a study on the degree of dizziness using the DHI questionnaire and classified it as mild (0-30), moderate (31-60), severe (61-100) in relation to performance in people with vestibular disorders [6]. The authors concluded that patients perceiving high handicap scores show greater functional impairment than patients with low handicap scores. In addition, another study reported an association between balance function and DHI in people with peripheral vestibular disorders, in which a high DHI score correlated with a high risk of falls [7]. The DHI itself is a widely used self-reporting questionnaire that has been utilized to evaluate dizziness, and has been translated into several languages with excellent psychometric validity, reliability and internal consistency in all translations [8-10]. In BPPV assessment specifically, the DHI showed $75 \%$ sensitivity and $92 \%$ negative predictive value (NPV) in reference to Dix-Hallpike tests [11].

Several studies have reported the correlation between vertigo, stress, and inflammation. In 2014, Gucluturk et al. [8] reported a link between inflammation and vertigo, in which they found increased pre-inflammatory mediators in patients with benign paroxysmal positional vertigo (BPPV) [12]. Other studies have also reported relationships between stress-related inflammation with the mechanism of peripheral vertigo [13, 14]. In a study on patients with vestibular neuritis, it was observed that the inflammatory process induced an increase in peripheral blood mononuclear cells that play a role in thrombotic processes in the tubular microvascular circulation, resulting in impaired vestibular function and manifesting as vertigo [14].

Several markers of inflammation have been evaluated in vertigo. The immune response to inflammation often has characteristic features, including an increase in the number of neutrophils and a decrease in the number of lymphocytes. A decrease in lymphocyte levels (lymphocytopenia) is a marker of a decline in the immune system, and occurs due to the redistribution of lymphocyte cells into the lymphatic system and accelerated apoptosis [15]. An example of early investigations into the dynamics of increasing neutrophil values and decreasing lymphocyte values reported that the severity of clinical status and clinical outcomes in $90 \mathrm{ICU}$ oncology patients correlated with the neutrophil to lymphocyte ratio (NLR), the intensity of neutrophilia, and lymphocytopenia [16]. The NLR is a frequently assessed marker in non-infectious inflammatory diseases, and is obtained by dividing the absolute or relative number of neutrophils by the absolute or relative number of lymphocytes. Although some variations exist, the normal range of NLR in a healthy adult population has been reported to be between 0.78 and 3.53 [17].

A study conducted by Ozbay et al. in 2014 reported an increase in the mean NLR value in peripheral vertigo patients compared to healthy controls [13]. In another study, it was reported that there was a two-fold increase in the mean NLR value in patients with vestibular neuritis $(3.31 \pm 2.02)$ relative to controls $(1.60 \pm 0.71)$, indicating that NLR values may reflect an inflammatory process and could be considered as a simple, reliable parameter to assess the severity of vestibular neuritis [14]. This was supported by another study, which concluded that increased levels of the NLR support the role of inflammation in patients with vestibular neuritis, although these inflammatory markers are not specific to support the diagnosis of vestibular neuritis [12].

The mechanisms behind NLR increase in peripheral vestibular disorders may be related to the physical and psychological stress that lead to increased expression of corticotropin releasing hormone (CRH). CRH overexpression stimulates sympathetic activity in the locus coeruleus of the brain stem by the release of catecholamines, and may result in symptoms such as pale, cold skin and vertigo $[18,19]$. Subsequent increase of parasympathetic activity then arises as a reciprocal inhibitory mechanism, which causes symptoms of nausea and vomiting. CRH overexpression also causes the release of cortisol through the hypothalamus-pituitary-adrenocortical axis (HPA axis), leading to an increase in neutrophil activation [20], a decrease in lymphocyte levels, and eventually resulting in higher NLR levels [21].

Although many studies have assessed the levels of NLR in peripheral vestibular vertigo, it is still unclear whether or not the NLR parameter is related to vertigo severity. Hence, this study aimed to assess the differences in the NLR levels of patients with peripheral vestibular vertigo of various DHI severity categories.

\section{Methods}

This cross-sectional study was conducted between July 2019 and March 2020 at the Wahidin Sudirohusodo Hospital and Hasanuddin University Teaching Hospital, Makassar. Prior to commencement, the study has received ethical approval from the Medical Ethics Committee of the Faculty of Medicine, Hasanuddin University (Ethics Number: 836/UN4.6.4.5.31/PP36/2019). The study population consisted of patients that present with peripheral vestibular vertigo, namely patients with complaints of spinning, nausea/vomiting, nystagmus and unsteadiness, aged over 18 years who underwent treatment in the Emergency Room (ER), neurology outpatient clinic, wards in Wahidin Sudirohusodo Hospital and Hasanuddin University Teaching Hospital, Makassar. 
Inclusion criteria were patients over 18 years of age with peripheral vestibular vertigo and were willing to participate in the study. The subjects were excluded if they had any of the following: complaints of fever, took steroids and non-steroidal anti-inflammatory drugs; on chemotherapy agents, smoke and/or consume alcohol, cardiovascular disease, cerebrovascular disease, and malignancy. All patients have provided informed consent prior to recruitment.

The measurement of neutrophil (\%) and lymphocyte (\%) was obtained during the vertigo attack on the first visit to the hospital. A sample of venous blood was collected and blood parameters were measured using the Sysmex XT-600i at the clinical laboratory of the Wahidin Sudirohusodo and the Hasanuddin University Teaching Hospital. After calculating the absolute lymphocyte and neutrophil value, the NLR was determined by dividing the absolute neutrophil value by the absolute lymphocyte value. After obtaining initial blood measurements, all patients underwent standard treatment and observation. Once the attacks have subsided, the patients were asked to complete the DHI questionnaire.

The results of this study were statistically analyzed using the Statistical Package for the Social Sciences (SPSS) 22.0 program (IBM, Chicago). The subjects were divided into the three DHI categories, and the ANOVA or Kruskal-Wallis test was implemented to assess differences in the lymphocyte count (\%), neutrophil count (\%) the NLR ratio, and mean age of the categories. A threshold of $p<0.05$ was used to define statistical significance.

\section{Results}

\section{Subject characteristics}

A total of 39 participants were recruited, consisting of 13 males and 26 females. As seen in Table 1, the mean age of the participants was $53.67 \pm 12.88$, with a minimum and maximum range of 20 and 73 years. The mean value of neutrophils was $65.07 \pm 12.21$ with a minimum and maximum value of 36.10 and 87.30 respectively.

Table 1 Characteristics of age, neutrophils, lymphocytes and neutrophil to lymphocyte ratio (NLR)

\begin{tabular}{lrrrrr}
\hline Characteristic & Mean & SD & Minimum & Maximum & Median \\
\hline Age & 53.67 & 12.88 & 20.00 & 73.00 & 58.00 \\
Neutrofil & 65.07 & 12.21 & 36.10 & 87.30 & 66.80 \\
Lymphocyte & 24.29 & 9.46 & 8.00 & 43.10 & 23.40 \\
NLR & 3.42 & 2.20 & 1.09 & 9.81 & 2.83 \\
\hline
\end{tabular}

The mean value of lymphocytes was $24.29 \pm 9.46$ with a minimum value of 8.00 and a maximum value of 43.10 . The mean value of NLR was $3.42 \pm 2.20$ with minimum value of 1.09 and maximum value of 9.81 .

\section{Gender-based differences in NLR}

Table 2 shows the comparison of age, neutrophils, lymphocytes and NLR based on gender. The mean age of male and female subjects were $56.23 \pm 13.81$ and $52.38 \pm 12.48$ respectively. No statistically significant difference was found between the mean age of the two genders $(p=0.375)$. There was also no statistically significant difference $(p=0.375)$ in the neutrophil value of male $(62.58 \pm 13.91)$ and female subjects $(66.31 \pm 11.35)$.

The lymphocyte count was also slightly higher in females $(25.01 \pm 9.57)$ relative to males $(22.85 \pm 9.45)$, although the difference was not statistically significant $(p=0.510)$. The mean NLR in males was $3.47 \pm 2.23$ and was $3.39 \pm 2.22$ in females. This NLR difference was not statistically significant $(p=0.858)$.

\section{Severity-based differences in NLR}

Subjects were then divided into 3 groups defined by the DHI. The measured parameters were then categorized based on the vertigo severity as measured by the DHI (Table 3). There was no significant difference $(p=0.524)$ in the mean age of subjects between the mild

Table 2 Comparative analysis of mean age, neutrophils, lymphocytes and neutrophil to lymphocyte ratio by gender

\begin{tabular}{lllll}
\hline Gender & Age & $\begin{array}{l}\text { Neutrophil } \\
\text { (\%) } \\
\text { Mean } \pm \text { SD }\end{array}$ & $\begin{array}{l}\text { Lymphocyte } \\
\text { (\%) }\end{array}$ & NLR \\
Mean \pm SD & Mean \pm SD \\
\hline Men & $56.23 \pm 13.81$ & $62.58 \pm 13.91$ & $22.85 \pm 9.45$ & $3.47 \pm 2.23$ \\
Women & $52.38 \pm 12.48$ & $66.31 \pm 11.35$ & $25.01 \pm 9.57$ & $3.39 \pm 2.22$ \\
$p$ value & $0.257^{* *}$ & $0.375^{*}$ & $0.510^{*}$ & $0.858^{* *}$ \\
\hline
\end{tabular}

${ }^{*}$-independent test, ${ }^{* *}$ Mann Whitney test, $N L R$ neutrophil to lymphocyte ratio

Table 3 Comparison of age, neutrophils, lymphocytes and neutrophil to lymphocyre ratio (NLR) by DHI (dizziness handicap inventory) category

\begin{tabular}{lllll}
\hline DHI & Age & $\begin{array}{l}\text { Neutrophil } \\
\text { (\%) } \\
\text { Mean } \pm \text { SD }\end{array}$ & $\begin{array}{l}\text { Lymphocyte } \\
\text { (\%) }\end{array}$ & NLR \\
Mean \pm SD & Mean \pm SD \\
\hline Mild & $51.00 \pm 13.39$ & $60.75 \pm 11.28$ & $29.97 \pm 9.46$ & $2.47 \pm 1.66$ \\
Moderate & $54.08 \pm 14.02$ & $62.91 \pm 10.98$ & $25.89 \pm 6.88$ & $2.64 \pm 0.96$ \\
Severe & $55.92 \pm 11.69$ & $71.55 \pm 12.38$ & $17.01 \pm 7.18$ & $5.15 \pm 2.59$ \\
$p$ value & $0.524^{* *}$ & $0.054^{*}$ & $0.001^{*}$ & $0.002^{* *}$ \\
\hline
\end{tabular}

*ANOVA test

**Kruskal Wallis Test 
$(51.00 \pm 13.39)$, moderate $(54.08 \pm 14.02)$, and severe $(55.92 \pm 11.69)$ categories. And whilst only a marginal difference $(p=0.054)$ was observed in the neutrophil levels of the three categories, there was a highly significant difference $(p=0.001)$ between the lymphocyte counts of the three categories. This leads to a significant difference $(p=0.002)$ in the NLR levels across the 3 severity categories (Fig. 1).

\section{Discussion}

Peripheral vestibular vertigo manifest as a collection of symptoms consisting of vertigo, nausea, vomiting, nystagmus and unsteadiness. About $80 \%$ of patients who experience vertigo report that the complaint interferes with their daily activities [4]. Several studies have reported the correlation between vertigo, stress and inflammation, such as increased pro-inflammatory mediators in BPPV, while others have reported the relation between stressrelated inflammation with the mechanism of peripheral vertigo $[13,14]$. Although the NLR is a convenient and commonly measured marker of inflammation, no studies have observed the inflammatory response across different severity levels of peripheral vestibular vertigo. This study aimed to assess whether differences exist between the NLR levels of patients with varying levels of severity as assessed by the DHI.

The main observation in this current study was that NLR values differed significantly between mild, moderate, and severe DHI. Whilst the NLR of mild and moderate DHI categories falls between the normal NLR range, the severe category exhibited an abnormally high NLR. The differences in NLR is likely attributed to lymphocyte variations, rather than variations in the neutrophil count. This is reflected by the variations in lymphocyte count between the three DHI categories, whilst no significant variance was observed between the neutrophil counts of the three categories. Despite the lack of significant difference in neutrophil counts however, it is worth noting that a higher trend of neutrophil value was observed in the severe DHI group.

This confirms the finding of previous studies that stipulate the connection between inflammatory processes with the occurrence of vertigo [13, 14]. As shown by Ozbay et al. a significant difference was found between the NLR levels of vertigo patients relative to healthy controls, while no significant difference was observed in neutrophil values [13]. However, the significantly higher lymphocyte count observed in this study are inconsistent with the previous literature [13, 14] which observed no significant difference between the lymphocyte count of a vertigo group with those of age- and sex-matched healthy controls. The differences between the current study and previous study could be related to certain methodological differences, such as the inclusion criteria and the blood measurement timeline wherein this study used the blood parameters obtained on the first visit during the vertigo attack. Aditionally, since this study emphasized on severity differences, this may indicate that lymphocytopenia may occur commonly in more severe forms of vertigo.

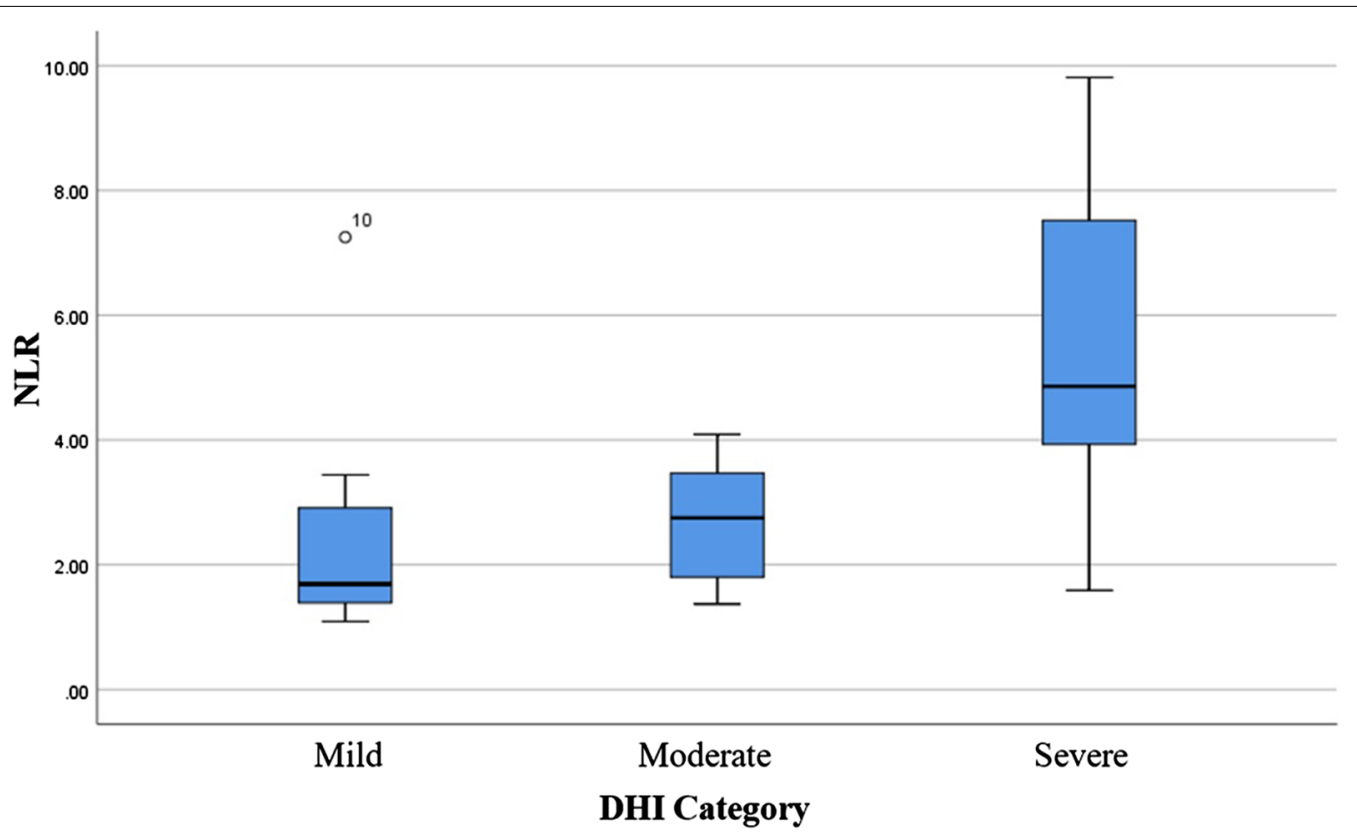

Fig. 1 Severity-dependent variations in the neutrophil to lymphocyte ratio (NLR) in peripheral vestibular vertigo. DHI dizziness handicap inventory 
A vicious cycle interaction can occur between somatic organic symptoms of vestibular vertigo and psychological stress. It can be assumed that although stress can cause vertigo attacks, vertigo can also cause stress. These two things are closely related. Vertigo attacks can be frightening to the patient because it can increase anxiety, thus worsening the emotional state of the patient. Anxiety and stress can cause various symptoms, one of which is through disorders of the autonomic nervous system [22], and inflammation. In this current study, we confirmed this phenomenon with the observation of a significant difference between the mean NLR of the three severity levels of vertigo.

In this study, no significant gender-based differences were observed on the neutrophil, lymphocyte and NLR parameters. The lack of gender-based difference of NLR has been observed in both healthy populations [23] and in other pathologies such as multiple sclerosis, wherein the investigators observed no significant difference in the NLR between the two genders [24].

Although the mean age of the mild, moderate, and severe DHI was not statistically significant, a higher agetrend was observed in the severe DHI category. Vertigo in the elderly is an increasing public health problem in most developing countries. In line with our finding, Vanspauwern et al. also showed that although not statistically significant, the relationship between age and DHI values were similar to this study [25]. Other studies have also shown that younger age groups with vertigo tend to have lower DHI values relative to older groups $[26,27]$. The difference in DHI between between the young and old age group, specifically the physical aspect, was even more discrepant in one study by Mann et al. [7]. This agebased difference in DHI is likely due to the decrease in postural stability among elderly populations [28].

A limitation of this study is that the samples were not categorized based on the classification of specific peripheral vestibular vertigo such as BPPV, vestibular neuritis, Meniere's disease and vestibular paroxysmia. In addition, only the baseline NLR was collected during a vertigo attack, while no samples were taken after recovery. Despite these limitations, this study is one of the first to analyze the NLR level in relation to the severity index of vertigo, and the results warrants further confirmation in a larger study.

\section{Conclusions}

In conclusion, a significant difference in the NLR was found between the three different vertigo severity groups, wherein NLR values rise with increase in vertigo severity. This warrants further exploration on the role of inflammatory biomarkers in vertigo pathophysiology and clinical assessment.
Abbreviations

NLR: Neutrophil to lymphocyte ratio; DHI: Dizziness Handicap Inventory.

\section{Acknowledgements}

We would like to thank all families who have participated in this study.

\section{Authors' contributions}

AKB conceptualized and supervised the study, drafted and finalized the manuscript. EIM performed the data collection and analysis. Both authors read and approved the final manuscript.

\section{Funding}

The study received no specific funding.

Availability of data and materials

All datasets generated during the study are available by request to the corresponding author.

\section{Declarations}

Ethical approval and consent to participate

This study was conducted according to the guidelines of the Declaration of Helsinki and was approved by the Ethics Committee of the Faculty of Medicine, Hasanuddin University.

\section{Consent for publications}

All subjects have provided consent for publication.

\section{Competing interests}

The authors declare no conflict of interest.

Received: 26 September 2021 Accepted: 28 November 2021

Published online: 13 December 2021

\section{References}

1. Baloh RW, Honrubia V, Kerber M Kevin A. Baloh and Honrubia's Clinical Neurophysiology of the Vestibular System, Fourth Edition. Los Angeles: Oxford University Press; 2011.

2. von Brevern M, Radtke A, Lezius F, Feldmann M, Ziese T, Lempert T, et al. Epidemiology of benign paroxysmal positional vertigo: a population based study. J Neurol Neurosurg Psychiatry.

3. de Moraes SA, de Souza Soares WJ, Rodrigues RAS, Fett WCR, Ferriolli E, Perracini MR. Dizziness in community-dewelling older adults: a population-based study. Braz J Otorhinolaryngol. 2011;77(6):691-9.

4. van Vugt VA, van der Horst HE, Payne RA, Maarsingh OR. Chronic vertigo: treat with exercise, not drugs. BMJ. 2017;j3727.

5. Jacobson GP, Newman CW. The development of the dizziness handicap inventory. Arch Otolaryngol Head Neck Surg. 1990;116(4):424-7.

6. Whitney SL, Wrisley DM, Brown KE, Furman JM. Is perception of handicap related to functional performance in persons with vestibular dysfunction? Otol Neurotol. 2004;25(2):139-43.

7. Mann GC, Whitney SL, Redfern MS, Borello-France DF, Furman JMR. Functional reach and single leg stance in patients with peripheral vestibular disorders. Neurol Rep. 1994;18(4):22.

8. Vereeck L, Truijen S, Wuyts F, Van de Heyning P. Test-retest reliability of the dutch version of the dizziness handicap inventory. B ENT. 2006;2(2):75-80.

9. Duracinsky M, Mosnier I, Bouccara D, Sterkers O, Chassany O, Working Group of the Société Française d'Oto-Rhino-Laryngologie (ORL). Literature review of questionnaires assessing vertigo and dizziness, and their impact on patients' quality of life. Value Health. 2007;10(4):273-84.

10. Jafarzadeh S, Bahrami E, Pourbakht A, Jalaie S, Daneshi A. Validity and reliability of the Persian version of the dizziness handicap inventory. J Res Med Sci. 2014;19(8):769-75.

11. Zamyslowska-Szmytke E, Politanski P, Jozefowicz-Korczynska M. Dizziness handicap inventory in clinical evaluation of dizzy patients. Int J Environ Res Public Health. 2021;18(5):2210. 
12. Güçlütürk MT, Ünal ZN, İsmi O, Çimen MBY, Ünal M. The role of oxidative stress and inflammatory mediators in benign paroxysmal positional vertigo. J Int Adv Otol. 2016;12(1):101.

13. Ozbay I, Kahraman C, Balikci HH, Kucur C, Kahraman NK, Ozkaya DP, et al. Neutrophil-to-lymphocyte ratio in patients with peripheral vertigo: a prospective controlled clinical study. Am J Otolaryngol. 2014;35(6):699-702.

14. Chung JH, Lim J, Jeong JH, Kim KR, Park CW, Lee SH. The significance of neutrophil to lymphocyte ratio and platelet to lymphocyte ratio in vestibular neuritis. Laryngoscope. 2015;125(7):E257-61.

15. Ayala A, Herdon C, Lehman D, Ayala C, Chaudry I. Differential induction of apoptosis in lymphoid tissues during sepsis: variation in onset, frequency, and the nature of the mediators. Blood. 1996;87(10):4261-75.

16. Zahorec R. Ratio of neutrophil to lymphocyte counts-rapid and simple parameter of systemic inflammation and stress in critically ill. Bratisl Lek Listy. 2001;102(1):5-14.

17. Forget P, Khalifa C, Defour J-P, Latinne D, Van Pel M-C, De Kock M. What is the normal value of the neutrophil-to-lymphocyte ratio? BMC Res Notes. 2017;10:12.

18. Peng S-Y, Zhuang Q-X, Zhang Y-X, Zhang X-Y, Wang J-J, Zhu J-N. Excitatory effect of norepinephrine on neurons in the inferior vestibular nucleus and the underlying receptor mechanism. J Neurosci Res. 2016;94(8):736-48.

19. De Cicco V, Tramonti Fantozzi MP, Cataldo E, Barresi M, Bruschini L, Faraguna $U$, et al. Trigeminal, visceral and vestibular inputs may improve cognitive functions by acting through the locus coeruleus and the ascending reticular activating system: a new hypothesis. Front Neuroanat. 2018;11(130):1-17.

20. Cannon JG, Fiatarone MA, Fielding RA, Evans WJ. Aging and stressinduced changes in complement activation and neutrophil mobilization. J Appl Physiol. 1994;76(6):2616-20.

21. Sherwood L. Human physiology: from cells to systems. Cengage learning; 2015.

22. Arruda-Olson AM, Reeder GS, Bell MR, Weston SA, Roger VL. Neutrophilia predicts death and heart failure after myocardial infarction: a communitybased study. Circ Cardiovasc Qual Outcomes. 2009;2(6):656-62.

23. Azab B, Camacho-Rivera M, Taioli E. Average values and racial differences of neutrophil lymphocyte ratio among a nationally representative sample of United States subjects. PLoS ONE. 2014;9(11):e112361-112361.

24. Al-Hussain F, Alfallaj MM, Alahmari AN, Almazyad AN, Alsaeed TK, Abdurrahman $\mathrm{AA}$, et al. Relationship between neutrophil-to-lymphocyte ratio and stress in multiple sclerosis patients. J Clin Diagn Res. 2017;11(5):1-4.

25. Vanspauwen R, Knoop A, Camp S, van Dinther J, Erwin Offeciers F, Somers $T$, et al. Outcome evaluation of the dizziness handicap inventory in an outpatient vestibular clinic. J Vestib Res. 2017;26(5-6):479-86.

26. Handa PR, Kuhn AMB, Cunha F, Schaffleln R, Ganança FF. Quality of life in patients with benign paroxysmal positional vertigo and/or Ménière's disease. Braz J Otorhinolaryngol. 2005;71(6):776-82.

27. Ten Voorde M, van der Zaag-Loonen HJ, van Leeuwen RB. Dizziness impairs health-related quality of life. Qual Life Res. 2011;21 (6):961-6

28. Baloh RW, Corona S, Jacobson KM, Enrietto JA, Bell T. A prospective study of posturography in normal older people. J Am Geriatr Soc. 1998:46(4):438-43.

\section{Publisher's Note}

Springer Nature remains neutral with regard to jurisdictional claims in published maps and institutional affiliations.

\section{Submit your manuscript to a SpringerOpen ${ }^{\circ}$ journal and benefit from:}

- Convenient online submission

- Rigorous peer review

- Open access: articles freely available online

- High visibility within the field

- Retaining the copyright to your article

Submit your next manuscript at $\boldsymbol{\nabla}$ springeropen.com 